\title{
Dynamic behaviors of a commensal symbiosis model with ratio-dependent functional response and one party can not survive independently
}

Runxin $\mathrm{Wu}^{*}$, Lin $\mathrm{Li}$

College of Mathematics and Physics, Fujian University of Technology, Fuzhou, Fujian, 350014, P. R. China.

\section{Abstract}

We propose a two-species commensal symbiosis model with ratio-dependent functional response

$$
\begin{aligned}
& \frac{d x}{d t}=x\left(-a_{1}-b_{1} x+\frac{c_{1} y}{x+y}\right), \\
& \frac{d y}{d t}=y\left(a_{2}-b_{2} y\right) .
\end{aligned}
$$

For autonomous case, we show that the unique positive equilibrium is globally stable if $a_{1}<c_{1}$ holds, and the boundary equilibrium $\left(0, \frac{a_{2}}{b_{2}}\right)$ is globally stable if $a_{1}>c_{1}$ holds. For nonautonomous case, some sufficient conditions which ensure the permanence and global attractivity of the system are obtained. Numeric simulations are carried out to show the feasibility of the main results. (c)2016 All rights reserved.

Keywords: Commensal symbiosis model, stability.

$2010 M S C:$ 34C25, 92D25, 34D20, 34D40.

\section{Introduction}

During the last decades, many scholars investigated the dynamic behaviors of the mutualism model or commensalism model, see [1, 2, 4, 5, 8-12, 14-17, 19-21, 23-29] and the references cited

*Corresponding author

Email addresses: runxinwu@163.com (Runxin Wu), dalaotu@163.com (Lin Li) 
therein. However, only recently did scholars paid attention to the commensal symbiosis model with one party can not survive independently (see [4, 5, 25]).

Based on the traditional two-species Lotka-Volterra model, Zhu et al. [29] proposed the following model:

$$
\begin{aligned}
& \dot{x}=x\left(a_{1}+b_{1} x+c_{1} y\right), \\
& \dot{y}=y\left(a_{2}+b_{2} y\right),
\end{aligned}
$$

where $a_{1}<0, a_{2}>0, b_{1}<0, b_{2}<0, c_{1}>0$. Here, $a_{1}<0$ means that the first species can not survive independently. Some qualitative analysis of the above system are carried out.

Yang et al. 25] argued that the non-autonomous case is more suitable, since the environment are change continuously, they investigated the dynamic behaviors of the following two-species commensalism model.

$$
\begin{aligned}
& \dot{x}=x\left(-a_{1}(t)-b_{1}(t) x+c_{1}(t) y\right), \\
& \dot{y}=y\left(a_{2}(t)-b_{2}(t) y\right),
\end{aligned}
$$

where $a_{1}(t), a_{2}(t), b_{1}(t), c_{1}(t), b_{2}(t)$ are all continuous functions bounded above and below by positive constants. Such topic as persistent, extinction and stability were investigated in [16].

Corresponding to system (1.2), Chen et al. [4, 5] proposed a discrete commensal symbiosis model

$$
\begin{aligned}
& x_{1}(k+1)=x_{1}(k) \exp \left\{-a_{1}(k)-b_{1}(k) x_{1}(k)+c_{1}(k) x_{2}(k)\right\}, \\
& x_{2}(k+1)=x_{2}(k) \exp \left\{a_{2}(k)-b_{2}(k) x_{2}(k)\right\} .
\end{aligned}
$$

They investigated the existence of positive $\omega$-periodic solution, the permanence, extinction and global attractivity of the system.

It brings to our attention that all of the above system made the assumption that the influence of the second species to the first one is linearized. Already, during the past two decades, in the study of predator-prey system, many scholars argued that in many conditions, especially when the predators have to search for food (consequently, have to share or compete for food), ratio-dependent functional response is more plausible, see [3, 6, 7, 13, 18, 22] and the references cited therein. Stimulated by the works of [3, 6, 7, 13, 18], in this paper, we study the following commensalism model with ratio-dependent functional response and one party can not survive independently:

$$
\begin{aligned}
& \frac{d x}{d t}=x\left(-a_{1}-b_{1} x+\frac{c_{1} y}{x+y}\right), \\
& \frac{d y}{d t}=y\left(a_{2}-b_{2} y\right) .
\end{aligned}
$$

Throughout this paper, we assume that $\left(\mathrm{H}_{1}\right)$ or $\left(\mathrm{H}_{2}\right)$ holds, here:

$\left(\mathrm{H}_{1}\right) a_{i}, b_{i}, i=1,2, c_{1}$ are all positive constants;

$\left(\mathrm{H}_{2}\right) a_{i}(t), b_{i}(t), i=1,2, c_{1}(t)$ are all continuous functions bounded above and below by some positive constants.

We arrange the paper as follows: In the next section, we investigate the existence and local stability property of the equilibria of system (1.3). In Section 3, we will investigate the global stability property of positive equilibrium of the system. In Section 4 , for the nonautonomous case, we obtain some sufficient conditions which ensure the permanence and global stability property of the system. In Section 5, two examples together with their numeric simulations are presented to show the feasibility of our main results. We end this paper by a brief discussion. 


\section{The existence and local stability of the equilibria}

The equilibria of system (1.3) is determined by the system

$$
\begin{aligned}
& x\left(-a_{1}-b_{1} x+\frac{c_{1} y}{x+y}\right)=0, \\
& y\left(a_{2}-b_{2} y\right)=0 .
\end{aligned}
$$

System (1.3) always admits the boundary equilibrium $A_{1}\left(0, \frac{a_{2}}{b_{2}}\right)$, also, if $a_{1}<c_{1}$, then system (1.3) admits a unique positive equilibrium $A_{2}\left(x^{*}, y^{*}\right)$, where

$$
x^{*}=\frac{-\left(a_{2} b_{1}+a_{1} b_{2}\right)+\sqrt{\left(a_{2} b_{1}+a_{1} b_{2}\right)^{2}-4\left(a_{1} a_{2}-a_{2} c_{1}\right) b_{1} b_{2}}}{2 b_{1} b_{2}}, \quad y^{*}=\frac{a_{2}}{b_{2}} .
$$

Obviously, $A_{2}\left(x^{*}, y^{*}\right)$ satisfies the equation

$$
\begin{gathered}
-a_{1}-b_{1} x^{*}+\frac{c_{1} y^{*}}{x^{*}+y^{*}}=0, \\
a_{2}-b_{2} y^{*}=0 .
\end{gathered}
$$

Concerned with the local stability property of the above three equilibria, we have

Theorem 2.1. $A_{1}\left(0, \frac{a_{2}}{b_{2}}\right)$ is unstable if $a_{1}<c_{1}$ and locally stable if $a_{1}>c_{1}$. Besides, if $A_{2}\left(x^{*}, y^{*}\right)$ exists, then it is locally stable.

Proof. The Jacobian matrix of the system 1.3 is calculated as

$$
J(x, y)=\left(\begin{array}{cc}
-a_{1}-2 b_{1} x+\frac{c_{1} y}{x+y}-\frac{c_{1} x y}{(x+y)^{2}} & \frac{c_{1} x^{2}}{(x+y)^{2}} \\
0 & -2 b_{2} y+a_{2}
\end{array}\right) .
$$

Then the Jacobian matrix of the system (1.3) about the equilibrium $A_{1}\left(0, \frac{a_{2}}{b_{2}}\right)$ is given by

$$
\left(\begin{array}{cc}
-a_{1}+c_{1} & 0 \\
0 & -a_{2}
\end{array}\right)
$$

The corresponding eigenvalues are $\lambda_{1}=-a_{1}+c_{1}, \lambda_{2}=-a_{2}<0$. Obviously, if $a_{1}>c_{1}$, then $\lambda_{1}<0$, in this case, $A_{2}\left(0, \frac{r_{2}}{a_{22}}\right)$ is locally stable, and $A_{1}\left(0, \frac{r_{2}}{a_{22}}\right)$ is unstable if $a_{1}<c_{1}$.

By using (2.1), the Jacobian matrix about the positive equilibrium $A_{2}$ is given by

$$
\left(\begin{array}{cc}
-b_{1} x^{*}-\frac{c_{1} x^{*} y^{*}}{\left(x^{*}+y^{*}\right)^{2}} & \frac{c_{1}\left(x^{*}\right)^{2}}{\left(x^{*}+y^{*}\right)^{2}} \\
0 & -a_{2}
\end{array}\right)
$$

The eigenvalues of the above matrix are $\lambda_{1}=-b_{1} x^{*}-\frac{c_{1} x^{*} y^{*}}{\left(x^{*}+y^{*}\right)^{2}}<0, \lambda_{2}=-a_{2}<0$.

Hence, $A_{2}\left(x^{*}, y^{*}\right)$ is locally stable. This ends the proof of Theorem 2.1.

\section{Global stability of the equilibria}

Since both boundary equilibrium and positive equilibrium are all possible locally stable, it is natural to find out suitable conditions which ensure the global stability property of the equilibria. 
Lemma $3.1([7])$. System

$$
\frac{d y}{d t}=y(a-b y)
$$

has a unique globally attractive positive equilibrium $y^{*}=\frac{a}{b}$.

Lemma 3.2. Assume that $\frac{c}{d}>a$, then system

$$
\frac{d y}{d t}=y\left(-a-b y+\frac{c}{y+d}\right)
$$

admits a unique positive equilibrium $y^{*}$ which is globally attractive, where $a, b, c$ are all positive constants.

Proof. Set $F(y)=-a-b y+\frac{c}{y+d}$. By simple computation, $F(y)=0$ has a unique positive solution

$$
y^{*}=\frac{1}{2} \frac{-b d-a+\sqrt{b^{2} d^{2}-2 a b d+a^{2}+4 b c}}{b} .
$$

Since $F(0)=-a+\frac{c}{d}>0$, and $F^{\prime}(y)=-\frac{b d^{2}+2 b d y+b y^{2}+c}{(y+d)^{2}}<0, F\left(y^{*}\right)=0$, it follows that

$$
F(y)>0 \text { for all } y \in\left(0, y^{*}\right)
$$

and

$$
F(y)<0 \text { for all } y \in\left(y^{*},+\infty\right) .
$$

Then it follows from Theorem 2.1 in [7] that $y^{*}$ is globally stable, i.e., $\lim _{t \rightarrow+\infty} y(t)=y^{*}$. This ends the proof of Lemma 3.2 .

Theorem 3.3. Assume that $a_{1}>c_{1}$ holds, then $A_{1}\left(0, \frac{a_{2}}{b_{2}}\right)$ is globally stable.

Proof. Noting that the second equation of 1.3 takes the form

$$
\frac{d y}{d t}=y\left(a_{2}-b_{2} y\right)
$$

By applying Lemma 3.1 to system (3.1), we know that system (3.1) has a unique globally attractive positive equilibrium $y^{*}=\frac{a_{2}}{b_{2}}$. i.e., $\lim _{t \rightarrow+\infty} y(t)=y^{*}$.

From the first equation of system (1.3), it immediately follows that

$$
\frac{d x}{d t} \leq x\left(-a_{1}+c_{1}\right)
$$

hence,

$$
x(t) \leq x(0) \exp \left\{\left(-a_{1}+c_{1}\right) t\right\} \rightarrow 0 \text { as } t \rightarrow+\infty .
$$

This ends the proof of Theorem 3.3 .

Theorem 3.4. Assume that $a_{1}<c_{1}$ holds, then $A_{2}\left(x^{*}, y^{*}\right)$ is globally stable. 
Proof. In the proof of Theorem 3.3, we had showed that $\lim _{t \rightarrow+\infty} y(t)=\frac{a_{2}}{b_{2}}$. That is, for any $\varepsilon>0$ small enough, there exists a $T>0$ such that for all $t>T$

$$
y^{*}-\varepsilon<y(t)<y^{*}+\varepsilon \text { for all } t>T \text {. }
$$

For $t>T$, it follows from the first equation of system $(1.3)$ and $(3.2)$ that

$$
\frac{d x}{d t} \leq x\left(-a_{1}-b_{1} x+\frac{c_{1}\left(y^{*}+\varepsilon\right)}{x+\left(y^{*}+\varepsilon\right)}\right) .
$$

Now let us consider the differential equation

$$
\frac{d u}{d t}=u\left(-a_{1}-b_{1} u+\frac{c_{1}\left(y^{*}+\varepsilon\right)}{u+\left(y^{*}+\varepsilon\right)}\right)
$$

since

$$
-a_{1}+\frac{c_{1}\left(y^{*}+\varepsilon\right)}{y^{*}+\varepsilon}=-a_{1}+c_{1}>0
$$

it follows from Lemma 3.2 that system (3.4) admits a unique global stable positive equilibrium

$$
u_{\varepsilon}^{*}=\frac{1}{2} \frac{-b_{1}\left(y^{*}+\varepsilon\right)-a_{1}+\sqrt{b_{1}^{2}\left(y^{*}+\varepsilon\right)^{2}-2 a_{1} b_{1}\left(y^{*}+\varepsilon\right)+a_{1}^{2}+4 b_{1} c_{1}\left(y^{*}+\varepsilon\right)}}{b_{1}} .
$$

By applying differential inequality theory to (3.3), it follows that

$$
\limsup _{t \rightarrow+\infty} x(t) \leq u_{\varepsilon}^{*}+\varepsilon \text {. }
$$

For $t>T$, from the first equation of system $(1.3)$ and $(3.2)$, we also have

$$
\frac{d x}{d t} \geq x\left(-a_{1}-b_{1} x+\frac{c_{1}\left(y^{*}-\varepsilon\right)}{x+\left(y^{*}-\varepsilon\right)}\right) .
$$

Now let us consider the differential equation

$$
\frac{d v}{d t}=v\left(-a_{1}-b_{1} v+\frac{c_{1}\left(y^{*}-\varepsilon\right)}{v+\left(y^{*}-\varepsilon\right)}\right)
$$

since

$$
-a_{1}+\frac{c_{1}\left(y^{*}-\varepsilon\right)}{y^{*}-\varepsilon}=-a_{1}+c_{1}>0,
$$

it follows from Lemma 3.2 that system 3.7 admits a unique global stable positive equilibrium

$$
v_{\varepsilon}^{*}=\frac{1}{2} \frac{-b_{1}\left(y^{*}-\varepsilon\right)-a_{1}+\sqrt{b_{1}^{2}\left(y^{*}-\varepsilon\right)^{2}-2 a_{1} b_{1}\left(y^{*}-\varepsilon\right)+a_{1}^{2}+4 b_{1} c_{1}\left(y^{*}-\varepsilon\right)}}{b_{1}} .
$$

By applying differential inequality theory to (3.6), it follows that

$$
\liminf _{t \rightarrow+\infty} x(t) \geq v_{\varepsilon}^{*}-\varepsilon .
$$

Setting $\varepsilon \rightarrow 0$ in 3.5 and $(3.8)$ leads to

$$
\lim _{t \rightarrow+\infty} x(t)=x^{*}
$$

where

$$
x^{*}=\frac{1}{2} \frac{-b_{1} y^{*}-a_{1}+\sqrt{b_{1}^{2}\left(y^{*}\right)^{2}-2 a_{1} b_{1} y^{*}+a_{1}^{2}+4 b_{1} c_{1} y^{*}}}{b_{1}} .
$$

This completes the proof. 


\section{Nonautonomous case}

Now let us consider the system

$$
\begin{aligned}
& \frac{d x}{d t}=x\left(-a_{1}(t)-b_{1}(t) x+\frac{c_{1}(t) y}{x+y}\right), \\
& \frac{d y}{d t}=y\left(a_{2}(t)-b_{2}(t) y\right),
\end{aligned}
$$

where $a_{i}(t), b_{i}(t), c_{1}(t), i=1,2$ are all continuous functions bounded above and below by positive constants. For the rest of the paper, for a bounded continuous function $g$ defined on $R$, let $g^{L}$ and $g^{M}$ be defined as

$$
g^{L}=\inf _{t \in R} g(t), \quad g^{M}=\sup _{t \in R} g(t) .
$$

Theorem 4.1. Assume that $c_{1}^{L}>a_{1}^{M}$ holds, then system 4.1) is permanent.

Proof. Let $(x(t), y(t))$ be any solution of system (4.1) with the initial conditions $x(0)>0, y(0)>0$. From the first equation of system (4.1) it follows that

$$
\dot{x}(t) \leq x\left(-a_{1}^{L}+c_{1}^{M}-b_{1}^{L} x\right) .
$$

Thus

$$
\limsup _{t \rightarrow+\infty} x(t) \leq \frac{-a_{1}^{L}+c_{1}^{M}}{b_{1}^{L}} \stackrel{\text { def }}{=} M_{1} .
$$

From the second equation of system (4.1), we have

$$
\dot{y}(t) \leq y\left(a_{2}^{M}-b_{2}^{L} y\right),
$$

thus,

$$
\limsup _{t \rightarrow+\infty} y(t) \leq \frac{a_{2}^{M}}{b_{2}^{L}} \stackrel{\text { def }}{=} M_{2}
$$

From the second equation of system (4.1), we have

$$
\dot{y}(t) \geq y\left(a_{2}^{L}-b_{2}^{M} y\right),
$$

thus,

$$
\liminf _{t \rightarrow+\infty} y(t) \geq \frac{a_{2}^{L}}{b_{2}^{M}} \stackrel{\text { def }}{=} m_{2}
$$

For any $\varepsilon>0$ small enough, it follows from 4.3 that there exists a $T>0$ such that

$$
y(t)>m_{2}-\varepsilon, \text { for all } t>T .
$$

From the first equation of system 4.1), it follows that

$$
\dot{x}(t) \geq x\left(-a_{1}^{M}-b_{1}^{M} x+\frac{c_{1}^{L}\left(m_{2}-\varepsilon\right)}{x+\left(m_{2}-\varepsilon\right)}\right) .
$$


Now let us consider the equation

$$
\dot{w}(t)=w\left(-a_{1}^{M}-b_{1}^{M} w+\frac{c_{1}^{L}\left(m_{2}-\varepsilon\right)}{w+\left(m_{2}-\varepsilon\right)}\right) .
$$

Since

$$
-a_{1}^{M}+\frac{c_{1}^{L}\left(m_{2}-\varepsilon\right)}{m_{2}-\varepsilon}=-a_{1}^{M}+c_{1}^{L}>0
$$

it follows from Lemma 3.2 that (4.4) admits a unique positive equilibrium

$$
w_{\varepsilon}^{*}=\frac{1}{2} \frac{-b_{1}^{M}\left(m_{2}-\varepsilon\right)-a_{1}^{M}+\sqrt{\Delta(\varepsilon)}}{b_{1}^{M}},
$$

where

$$
\Delta(\varepsilon)=\left(b_{1}^{M}\right)^{2}\left(m_{2}-\varepsilon\right)^{2}-2 a_{1}^{M} b_{1}^{M}\left(m_{2}-\varepsilon\right)+\left(a_{1}^{M}\right)^{2}+4 b_{1}^{M} c_{1}^{M}\left(m_{2}-\varepsilon\right),
$$

which is globally stable. Thus

$$
\liminf _{t \rightarrow+\infty} x(t) \geq w_{\varepsilon}^{*}-\varepsilon .
$$

Setting $\varepsilon \rightarrow 0$, it immediately follows that

$$
\liminf _{t \rightarrow+\infty} x(t) \geq m_{1}
$$

where

$$
m_{1}=\frac{1}{2} \frac{-b_{1}^{M} m_{2}-a_{1}^{M}+\sqrt{\left(b_{1}^{M}\right)^{2} m_{2}^{2}-2 a_{1}^{M} b_{1}^{M} m_{2}+\left(a_{1}^{M}\right)^{2}+4 b_{1}^{M} c_{1}^{M} m_{2}}}{b_{1}^{M}} .
$$

This ends the proof of Theorem 4.1.

Before we state the stability property of this section, we introduce some notations. Set

$$
\begin{aligned}
& A_{1}(t) \stackrel{\text { def }}{=} b_{1}(t)-\frac{c_{1}(t) M_{2}}{\left(m_{1}+m_{2}\right)^{2}}, \\
& A_{2}(t) \stackrel{\text { def }}{=} b_{2}(t)-\frac{c_{1}(t) M_{1}}{\left(m_{1}+m_{2}\right)^{2}} .
\end{aligned}
$$

Theorem 4.2. Assume that $c_{1}^{L}>a_{1}^{M}$, assume further that

$$
\liminf _{t \rightarrow+\infty}\left\{A_{1}(t), A_{2}(t)\right\}>0
$$

then for any positive solutions $(x(t), y(t))$ and $\left(x_{1}(t), y_{1}(t)\right)$ of system (4.1), one has

$$
\lim _{t \rightarrow+\infty}\left(\left|x(t)-x_{1}(t)\right|+\left|y(t)-y_{1}(t)\right|\right)=0 .
$$

Proof. Condition (4.5) implies that there exists a positive constant $\varepsilon$ small enough (without loss of generality, we may assume that $\left.\varepsilon<\frac{1}{2}\left\{m_{1}, m_{2}\right\}\right)$ and large enough $T_{1}>0$ such that 


$$
\begin{aligned}
& A_{1}(\varepsilon, t) \stackrel{\text { def }}{=} b_{1}(t)-\frac{c_{1}(t)\left(M_{2}+\varepsilon\right)}{\left(m_{1}+m_{2}-2 \varepsilon\right)^{2}} \geq \varepsilon, \\
& A_{2}(\varepsilon, t) \stackrel{\text { def }}{=} b_{2}(t)-\frac{c_{1}(t)\left(M_{1}+\varepsilon\right)}{\left(m_{1}+m_{2}-2 \varepsilon\right)^{2}} \geq \varepsilon .
\end{aligned}
$$

For two arbitrary positive solutions $(x(t), y(t))^{T}$ and $\left(x_{1}(t), y_{1}(t)\right)^{T}$ of system (4.1). For above $\varepsilon>0$, it follows from Theorem 4.1 that there exists a $T>T_{1}$, such that for all $t \geq T$,

$$
\begin{array}{ll}
x(t), x_{1}(t)<M_{1}+\varepsilon, & y(t), y_{1}(t)<M_{2}+\varepsilon, \\
x(t), x_{1}(t)>m_{1}-\varepsilon, & y(t), y_{1}(t)>m_{2}-\varepsilon .
\end{array}
$$

Now we let

$$
V(t)=\left|\ln x(t)-\ln x_{1}(t)\right|+\left|\ln y(t)-\ln y_{1}(t)\right| .
$$

Then for $t>T$, we have

$$
\begin{aligned}
D^{+} V(t) \leq & \operatorname{sgn}\left(x(t)-x_{1}(t)\right)\left(-b_{1}(t) x(t)+\frac{c_{1}(t) y(t)}{x(t)+y(t)}+b_{1}(t) x_{1}(t)-\frac{c_{1}(t) y_{1}(t)}{x_{1}(t)+y_{1}(t)}\right) \\
& +\operatorname{sgn}\left(y(t)-y_{1}(t)\right)\left(-b_{2}(t) y(t)+b_{2}(t) y_{1}(t)\right) \\
\leq & -b_{1}(t)\left|x(t)-x_{1}(t)\right|+c_{1}(t)\left|\frac{y(t)}{x(t)+y(t)}-\frac{y_{1}(t)}{x_{1}(t)+y_{1}(t)}\right|-b_{2}(t)\left|y(t)-y_{1}(t)\right| \\
\leq & -\left(b_{1}(t)-\frac{c_{1}(t)\left(M_{2}+\varepsilon\right)}{\left(m_{1}+m_{2}-\varepsilon\right)^{2}}\right)\left|x(t)-x_{1}(t)\right| \\
& -\left(b_{2}(t)-\frac{c_{1}(t)\left(M_{1}+\varepsilon\right)}{\left(m_{1}+m_{2}-\varepsilon\right)^{2}}\right)\left|y(t)-y_{1}(t)\right| .
\end{aligned}
$$

From (4.7), by using (4.6), similar to the analysis of (3.6)-(3.8) in [22], one can conclude that

$$
\lim _{t \rightarrow+\infty}\left[\left|x(t)-x_{1}(t)\right|+\left|y(t)-y_{1}(t)\right|\right]=0 .
$$

This ends the proof of the Theorem 4.2 .

\section{Numeric simulations}

Now let us consider the following example.

Example 5.1. Consider the following system

$$
\begin{aligned}
& \frac{d x}{d t}=x\left(-a_{1}-x+\frac{y}{x+y}\right), \\
& \frac{d y}{d t}=y(1-y) .
\end{aligned}
$$

In this system, corresponding to system (1.3), we take $b_{1}=c_{1}=a_{2}=b_{2}=1$.

(1) Now take $a_{1}=2$, then $a_{1}>c_{1}$, it follows from Theorem 3.3 that $(0,1)$ is globally stable. Numeric simulation (Fig. 1) support this assertion.

(2) Now take $a_{1}=\frac{1}{2}$, then $a_{1}<c_{1}$, it follow from Theorem 3.4 that the unique positive equilibrium $(0.281,1)$ is globally stable. Numeric simulation (Fig. 2) support this assertion. 


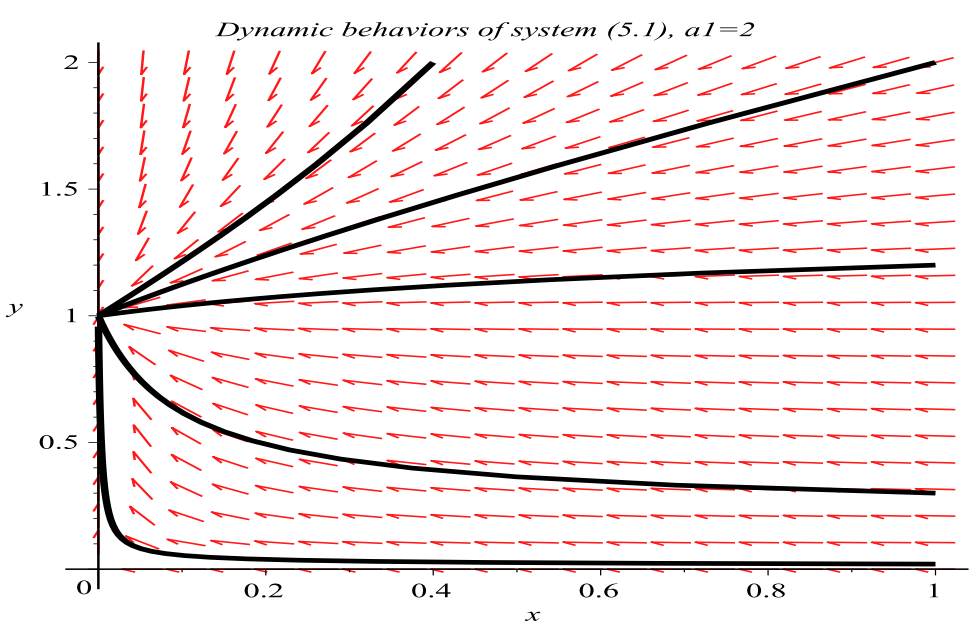

Figure 1: Numeric simulations of system (5.1) with $a_{1}=2$ and the initial conditions $(x(0), y(0))=(0.4,2),(1,0.3),(1,0.02),(1,0.3)$ and $(1,1.2)$, respectively.

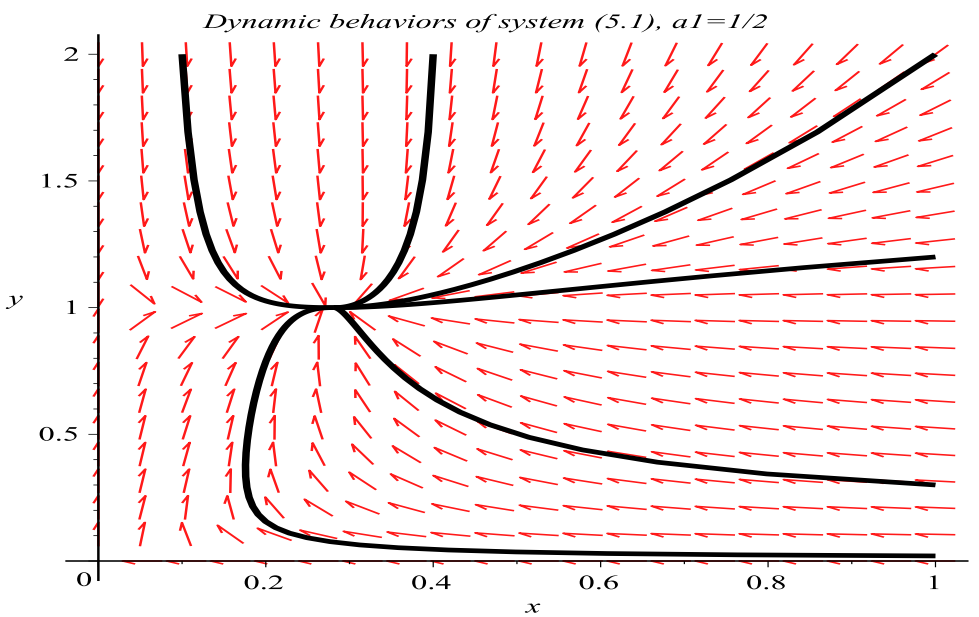

Figure 2: Numeric simulations of system (5.1) with $a_{1}=\frac{1}{2}$ and the initial conditions $(x(0), y(0))=(0.4,2),(1,0.3),(1,0.02),(1,0.3),(1,1.2)$ and $(0.1,2)$, respectively.

\section{Example 5.2.}

$$
\begin{aligned}
& \frac{d x(t)}{d t}=x(t)\left(-2+\cos (t)-x(t)+\frac{\left(3+\frac{1}{t^{2}+1}\right) y(t)}{x(t)+y(t)}\right), \\
& \frac{d y(t)}{d t}=y(t)\left(5+\cos (t)-\left(3+\frac{1}{2} \sin (t)\right) y(t)\right) .
\end{aligned}
$$

Corresponding to system (4.1), one has

$$
a_{1}(t)=2-\cos (t), \quad c_{1}(t)=3+\frac{1}{t^{2}+1},
$$




$$
a_{2}(t)=5+\cos (t), \quad b_{2}(t)=3+\frac{1}{2} \sin (t)
$$

Obviously, $c_{1}^{L}>a_{1}^{M}$, hence, the conditions of Theorem 4.1 hold, it follows from Theorem 4.1 that system (5.2) is permanent. Numeric simulations (Figs. 3 and 4) also support this assertion.

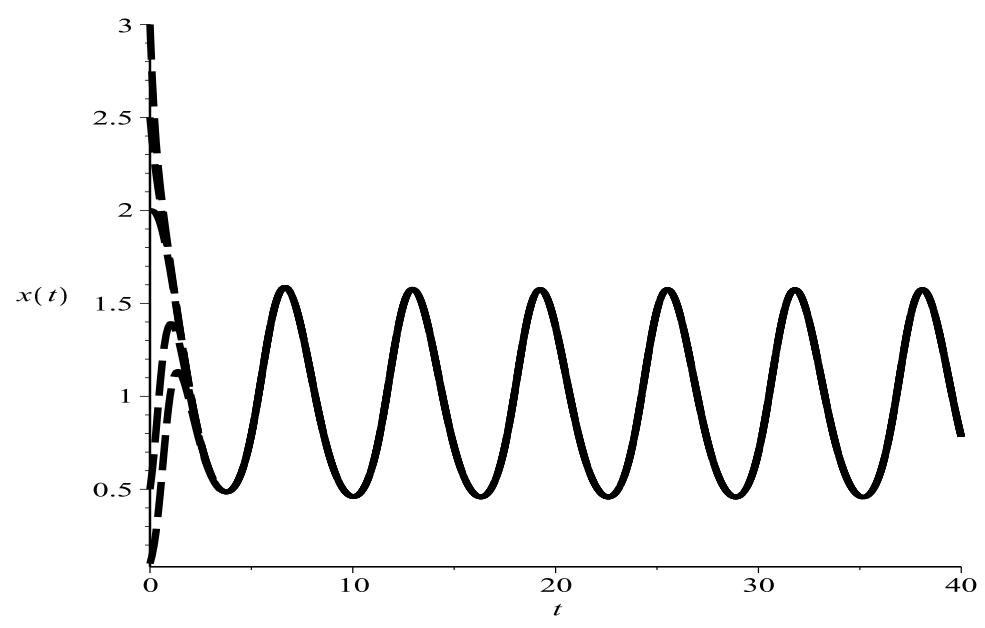

Figure 3: Dynamics behavior of the first species in system 5.2 with the initial conditions $(x(0), y(0))=(0.1,1),(2,2),(3,3),(2.5,2.5)$, and $(0.5,0.5)$, respectively.

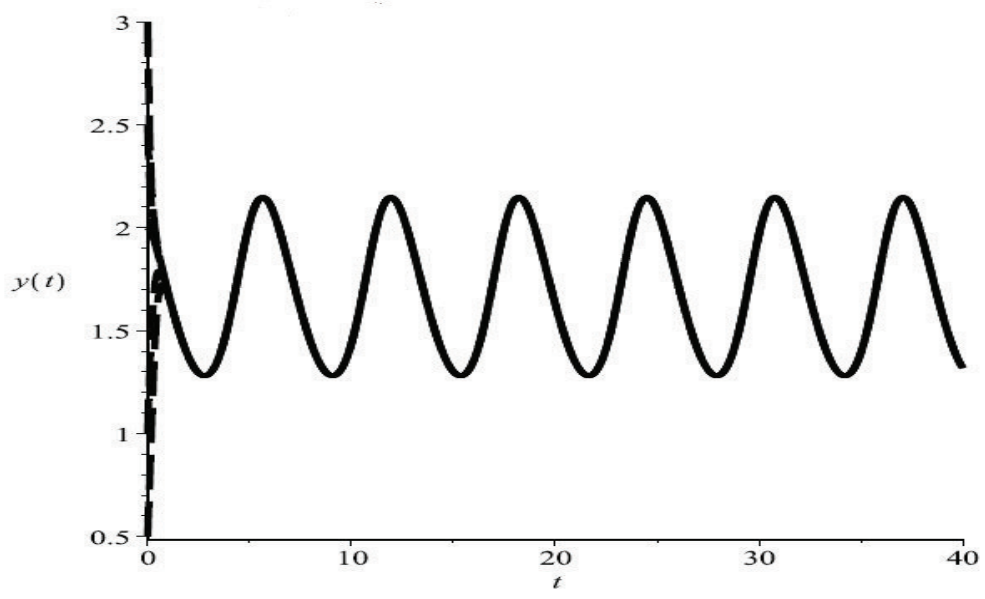

Figure 4: Dynamics behavior of the second species in system 5.2 with the initial conditions $(x(0), y(0))=(0.1,1),(2,2),(3,3),(2.5,2.5)$, and $(0.5,0.5)$, respectively.

\section{Conclusion}

We propose a two-species commensal symbiosis model with ratio-dependent functional response and one party can not survive independently. We show that for the autonomous case, if $a_{1}>c_{1}$, 
that is, the intrinsic death rate of the first species is larger than the commensalism effect between the species, then the first species will be driven to extinction, and if $a_{1}<c_{1}$, that is, the cooperative effect between two species is large than the intrinsic death rate of the first species, then two species could be coexist in a stable state.

For the nonautonomous case, we show that under the condition $a_{1}^{M}<c_{1}^{L}$, two species can be persistent. However, we are not able to show that this condition enough to guarantee the global stability of the system, indeed, we need some extra conditions (condition (4.5)) to ensure the global stability property of the system.

We mention here that a suitable system should incorporate some past state of the species, and this leads to a system with time delay, we will leave this for future investigation.

\section{Acknowledgment}

The research was supported by the Natural Science Foundation of Fujian Province (2015J01012, 2015J01019, 2015J05006) and the Scientific Research Foundation of Fuzhou University (XRC-1438).

\section{References}

[1] F. D. Chen, Permanence for the discrete mutualism model with time delays, Math. Comput. Modelling, 47 (2008), 431-435. 1

[2] L. J. Chen, L. J. Chen, Z. Li, Permanence of a delayed discrete mutualism model with feedback controls, Math. Comput. Modelling, 50 (2009), 1083-1089. 1

[3] F. D. Chen, Z. Li, Y. J. Huang, Note on the permanence of a competitive system with infinite delay and feedback controls, Nonlinear Anal. Real World Appl., 8 (2007), 680-687. 1

[4] F. D. Chen, C. T. Lin, L. Y. Yang, On a discrete obligate Lotka-Volterra model with one party can not surivive independently, Journal of Shenyang University (Natural Science), 4 (2015), 336-338. 11, 1

[5] F. D. Chen, L. Q. Pu, L. Y. Yang, Positive periodic solution of a discrete obligate Lotka-Volterra model, Commun. Math. Biol. Neurosci., 2015 (2015), 9 pages. 1, 1

[6] F. D. Chen, J. L. Shi, On a delayed nonautonomous ratio-dependent predator-prey model with Holling type functional response and diffusion, Appl. Math. Comput., 192 (2007), 358-369. 1

[7] L. S. Chen, X. Y. Song, Z. Y. Lu, Mathematical models and methods in ecology, (Chinese) Shichuan Science and Technology Press, Chengdu, (2002). 1, 3.1, 3

[8] L. J. Chen, X. D. Xie, Permanence of an N-species cooperation system with continuous time delays and feedback controls, Nonlinear Anal. Real World Appl., 12 (2001), 34-38. 1

[9] F. D. Chen, X. D. Xie, Study on the dynamic behaviors of cooperation population modeling, (Chinese) Science Press, Beijing, (2014).

[10] L. J. Chen, X. D. Xie, L. J. Chen, Feedback control variables have no influence on the permanence of a discrete $N$-species cooperation system, Discrete Dyn. Nat. Soc., 2009 (2009), 10 pages.

[11] F. D. Chen, X. D. Xie, X. F. Chen, Dynamic behaviors of a stage-structured cooperation model, Commun. Math. Biol. Neurosci., 2015 (2015), 19 pages.

[12] F. D. Chen, J. H. Yang, L. J. Chen, X. D. Xie, On a mutualism model with feedback controls, Appl. Math. Comput., 214 (2009), 581-587. 1

[13] M. Fan, K. Wang, Periodicity in a delayed ratio-dependent predator-prey system, J. Math. Anal. Appl., 262 (2001), 179-190. 1

[14] Y. K. Li, T. W. Zhang, Permanence of a discrete $n$-species cooperation system with time-varying delays and feedback controls, Math. Comput. Modelling, 53 (2011), 1320-1330. 1

[15] Z. J. Liu, J. H. Wu, R. H. Tan, Y. P. Chen, Modeling and analysis of a periodic delayed two-species model of facultative mutualism, Appl. Math. Comput., 217 (2010), 893-903.

[16] Z. S. Miao, X. D. Xie, L. Q. Pu, Dynamic behaviors of a periodic Lotka-Volterra commensal symbiosis model with impulsive, Commun. Math. Biol. Neurosci., 2015 (2015), 15 pages. 1

[17] R. X. Wu, L. Lin, X. Y. Zhou, A commensal symbiosis model with Holling type functional response, J. Math. Computer Sci., 16 (2016), 364-371. 1

[18] D. M. Xiao, W. X. Li, M. A. Han, Dynamics in a ratio-dependent predator-prey model with predator harvesting, J. Math. Anal. Appl., 324 (2006), 14-29. 1 
[19] X. D. Xie, F. D. Chen, Y. L. Xue, Note on the stability property of a cooperative system incorporating harvesting, Discrete Dyn. Nat. Soc., 2014 (2014), 5 pages. 1

[20] X. D. Xie, F. D. Chen, K. Yang, Y. L. Xue, Global attractivity of an integrodifferential model of mutualism, Abstr. Appl. Anal., 2014 (2014), 6 pages.

[21] X. D. Xie, Z. S. Miao, Y. L. Xue, Positive periodic solution of a discrete Lotka-Volterra commensal symbiosis model, Commun. Math. Biol. Neurosci., 2015 (2015), 10 pages. 1

[22] X. D. Xie, Y. L. Xue, J. H. Chen, T. T. Li, Permanence and global attractivity of a nonautonomous modified Leslie-Gower predator-prey model with Holling-type II schemes and a prey refuge, Adv. Difference Equ., 2016 (2016), 11 pages. 1, 4

[23] Y. L. Xue, F. D. Chen, X. D. Xie, R. Y. Han, Dynamic behaviors of a discrete commensalism system, Ann. Appl. Math., 31 (2015), 452-461. 1

[24] Y. L. Xue, X. D. Xie, F. D. Chen, R. Y. Han, Almost periodic solution of a discrete commensalism system, Discrete Dyn. Nat. Soc., 2015 (2015), 11 pages.

[25] L. Y. Yang, R. Y. Han, Y. L. Xue, F. D. Chen, On a Nonautonomous Obligate Lotka-Volterra Model, J. Sanming Univ., 6 (2014), 15-18. 1, 1

[26] W. S. Yang, X. P. Li, Permanence of a discrete nonlinear N-species cooperation system with time delays and feedback controls, Appl. Math. Comput., 218 (2011), 3581-3586.

[27] K. Yang, Z. S. Miao, F. D. Chen, X. D. Xie, Influence of single feedback control variable on an autonomous Holling-II type cooperative system, J. Math. Anal. Appl., 435 (2016), 874-888.

[28] K. Yang, X. D. Xie, F. D. Chen, Global stability of a discrete mutualism model, Abstr. Appl. Anal., 2014 (2014), 7 pages.

[29] Z. F. Zhu, Y. A. Li, F. Xu, Mathematical analysis on commensalism Lotka-Volterra model of populations, Chongqing Institute of Technology (Natural Science), 21 (2007), 59-62. 1 